\title{
Subject Index Vol. 26, 1997
}

Accidental injury 160 Accumulator 236 Acrylic resin 33 Amebiasis 107 Anomaly 214

Anterior cerebral artery occlusion 93 Arachnoid cyst(s) 48,312 Associated abnormalities 304 Astrocytoma, anaplastic 97 -, low grade 36 Atlas 214

Balamuthia mandrillaris 107 Birth injury 269 Brain damage 269

neoplasm 315

tumor(s) 25,68

ventricles 185

Cavernous angioma 322 Cerebral blood flow 200

infarction 93

sparganosis, child 103 Cerebrospinal fluid 185

- diversionary devices

236

- shunt 115,190

- - infection rates 180 Chiari malformation 57,288

I malformation 190 Children 8,83,261 Compartment syndrome 160 Compliance 236 Complications, cranioplasty 33 Computed tomography 214 Congenital anomaly 185 kyphosis 130 Conus medullaris 2 Cortical mapping 144

stimulation 68 Cosmetic deformity 304 Cranial vault expansion 296 Craniopharyngioma, pediatric

255 Cranioplasty 33 Craniosynostosis 288 , 296 Cyanoacrylate 120 
Diastematomyelia 17 Duane retraction syndrome

157

Echinococcosis 208 Enzyme-linked immunosorbent

assay 103 Ependymoma 157,322 Epicranial fluid 160 Epilepsy 83, 144 Evoked potentials 120 External beam radiation

therapy 322 Extra-axial fluid 160

Facial trauma 261 Fall 160

Filum terminale 2, 8 Frameless stereotaxy 68, 127 Free radicals 120 Functional magnetic resonance imaging 68

raphy 144

Glioma 25

-, brainstem 36

-, brainstem 36

Hair, shaving 180 Head injury 200,261

-

Hindbrain herniation 288

Histiocytosis 315

Hydrocephalus 107, 127, 197,

236,269,288,296 Hydromyelia 57

In vivo proton MR spectros-

copy 93 Infant carriers 304 Infants 25,261 Infection shunt 115 Intracranial aneurysm 41

hydatidcyst 208

hypertension 296

pressure 288 
Intraoperative monitoring 247 Intraventricular hemorrhage 281

Lambdoid molding 304 Laminectomy 190 Lesions 83 Lipoma 8 Lipomyelomeningocele 8

Magnetic resonance imaging

103 Medulloblastoma 322 Meningoencephalitis 107 Methyl methacrylate 33 Middle fossa 48,312 Molecular biology 36 Motor evoked potentials 120,

247 Mouse embryos 185 Myelomeningocele 57 Myelopathy 214 Myxopapillary ependymoma 2

Natural history, arachnoid

cysts 48 Neural tube defects 17 Neurobehavior 255 Neurocysticercosis 93 Neuroendoscopy 127 Neurophysiology 247

Orbital fracture 261 Outcome, neurobehavioral 255 Overdrainage 236

Pineoblastoma 36 Positional molding 304 Positron emission tomography

144 Posterior circulation 41 Posthemorrhagic hydrocephalus 281 Prematurity 281 Presurgical evaluation 144

Seizure(s) 68 - disorder 144 -, pediatric 315 
Shunt 115,127

malfunction 236

SIIDS 304

Skin preparation for surgery 180

Somatosensory evoked potentials 120

Spinabifíida 57,214

- aperta 185 Spinal canal stenosis 214

cord tumor, pediatric 247

dysraphism 17 Spine 130,214 Spontaneous bacterial peritonitis 115

resolution 312

thrombosis 41 Starling resistor 236 Stereotactic surgery 315 Suboccipital craniectomy 190 Surgery 83

Surgical stabilization 130

Surgically induced neural tube

defects 185 Survival, long-term 97 Syringomyelia 190

Temporal lobe agenesis 48

- resections 83 Tethered cord 8

- syndrome 120 Tonsillar herniation 296 Torticollis 304 Treatment 57

Urokinase 281

Vacuum extraction 269 Ventriculo-gallbladder

197 Ventriculoperitoneal shunts

236

Xanthogranuloma 315 Xenon computed tomography 200

\section{KARGER}

Fax+41 613061234 E-Mail karger@karger.ch www.karger.com 
